\title{
Campos profesionales y mercados laborales (perspectivas de los egresados)
}

\author{
Claudia Benassini Félix ${ }^{1}$
}

\section{Antecedentes}

\begin{abstract}
A
PRINCIPIOS de la década, las escuelas de comunicación en México comenzaron a plantearse la pertinencia de realizar seguimientos de egresados que arrojaran un diagnóstico de la situación del campo de la comunicación, que eventualmente incidiera en la planeación curricular. En ese momento la Universidad Iberoamericana-planteles Santa Fe y León, el Instituto Tecnológico y de Estudios Superiores de Occidente y la Universidad Autónoma del Estado de México, entre otras instituciones, concluyeron investigaciones al respecto.

A partir de 1993 el tema volvió a formar parte de la agenda del Consejo Nacional para la Enseñanza y la Investigación de las Ciencias de la Comunicación (CONEICC). Incluso se planteó la pertinencia de llevar a cabo una encuesta nacional sobre prácticas profesionales en comunicación, a partir de la cual se elaboró la primera propuesta. Prácticamente al mismo tiempo la Federación Latinoamericana de Asociaciones de Facultades de Comunicación Social (Felafacs) lanzó una convocatoria a las asociaciones nacionales afiliadas, para participar en un proyecto latinoamericano de características similares al emprendido por el CONEICC.

Aceptada la propuesta, México se integró al proyecto de realizar un estudio comparativo integral de las expectativas y las tendencias de las actividades profesionales en el campo de la comunicación, tomando en cuenta las diversas perspectivas: académica, laboral, empresarial y social.
\end{abstract}

1. Universidad Iberoamericana, Departamento de Comunicación. 


\section{Objetivos}

Las finalidades que orientan esta fase de la investigación son congruentes con las que se plantearon en el proyecto latinoamericano sobre campos profesionales y mercados laborales: conocer el punto de vista de los egresados de las escuelas de comunicación, para identificar tanto las perspectivas del mercado laboral como las necesidades de formación académica. Es decir, se trata de un acercamiento a sus actividades profesionales, así como a la percepción que tienen sobre la formación recibida, y las de posibles proyecciones y necesidades de su actividad.

Como en el resto de los países latinoamericanos en México, esta etapa tiene diversas utilidades. En primer lugar, la realización o actualización de directorios de profesionales de las facultades que participan en la investigación. En segundo lugar, la obtención de información que podrá aprovecharse tanto a nivel particular - por cada institución que se integre a la investigación- como a nivel general pues, entre otros usos, permitirá la realización de un primer diagnóstico nacional de la situación de los egresados de las escuelas de comunicación en México.

\section{Método de trabajo}

Se integró un equipo nacional conformado por un coordinador general y un representante de cada una de las instituciones interesadas en participar en la investigación. Este equipo asistió a dos talleres en los que se llevaron a cabo tres actividades principales:

- La reelaboración del cuestionario previamente realizado por el equipo de Felafacs, con la finalidad de adaptarlo a las necesidades nacionales. Después, los representantes institucionales hicieron una segunda reelaboración para contar con un diagnóstico más profundo de la situación de sus egresados.

- El acuerdo sobre los criterios para seleccionar una muestra representativa que fuera de utilidad tanto para el estudio institucional como para el nacional. Sin embargo, en algunos casos fue prácticamente imposible tomar este criterio al pie de la letra. Por lo tanto, parte de los representantes institucionales se vieron obligados a efectuar ciertos ajustes, en función de la disponibilidad de los egresados localizables.

- El método de captura de datos para la integración de una base de datos nacional. Esta etapa de los talleres incluyó la capacitación en el manejo del programa adecuado y la elaboración de un manual de captura que fue distribuido entre los participantes en el estudio. 
Los resultados presentados en este trabajo corresponden a la primera fase de levantamiento de datos de la encuesta nacional aplicada a los egresados de escuelas de comunicación. La información todavía tiene carácter preliminar, pues a la fecha diversas instituciones continúan entregando información que alimenta la base de datos. Por lo tanto y con el propósito de mostrar tendencias, este primer reporte se presenta en porcentajes.

\section{Las instituciones participantes}

Los talleres organizados para la metodología y composición del cuestionario para egresados contaron con la presencia de 20 instituciones pioneras, a las que se han añadido otras. Este reporte proporciona los datos de ocho: Escuela de Comunicación Social de Sinaloa, Escuela Nacional de Estudios Profesionales Acatlán, Instituto Tecnológico de Estudios Superiores de Monterrey-campus Morelos, Universidad de las Américas-Puebla, Universidad Autónoma Metropolitana-Xochimilco, Universidad Iberoamericana-planteles Laguna y Santa Fe y Universidad Autónoma del Estado de México. Asimismo, otras instituciones han generado reportes individuales, los cuales se sumarán a la base de datos ${ }^{2}$. Por concluirse en el transcurso de este año. Por lo que se refiere al carácter de la institución, el cuadro 1 sintetiza la información al respecto:

\section{CUADRO 1}

Carácter de la institución de la que proceden los egresados participantes en la encuesta

\begin{tabular}{|l|c|}
\hline Carácter de la institución & $\%$ \\
\hline Pública & 54.8 \\
\hline Privada & 45.2 \\
\hline Total & 100.0 \\
\hline
\end{tabular}

Los datos que se presentan en este cuadro coinciden con los arrojados en el cuestionario institucional. ${ }^{3}$ Por lo tanto, puede afirmarse que a pesar de que el aumento de las escuelas de comunicación ha sido en el sector privado, las

2. Por ejemplo, la UNAM y la Universidad de Sonora han publicado sus respectivos reportes. Por razones fundamentalmente de carácter tecnológico, estos datos no se han podido incluir en la base, aunque se considerarán en el siguiente reporte, una vez que esté concluida toda la información nacional. Asimismo, la Escuela de Periodismo Carlos Septién García - una de las primeras en concluir la investigación-presentó un informe más actualizado, que también se incluirá en el siguiente reporte.

3. Véase Claudia Benassini, (comp.). ¿Desde dónde se enseña la comunicación en México? primer reporte de trabajo del equipo Campos Profesionales y Mercados Laborales, UIA, Mex., 1996. 
universidades públicas - numéricamente inferiores - son las que concentran el mayor número de estudiantes. Sobresalen la Universidad Nacional Autónoma de México (con una población aproximada de seis mil estudiantes de comunicación ubicados en sus tres planteles) y la Universidad Autónoma de Nuevo León (con una población aproximada de 2,500 estudiantes de comunicación). Tal como se reportó en la primera etapa, estas cifras repercuten en la formación de los futuros profesionales, tanto por la disponibilidad de profesores como por la dotación de equipo para prácticas de taboratorio. Asimismo, como se verá más adelante, estos problemas repercuten en la evaluación que los egresados hacen de su institución.

\section{Características de la muestra}

Como ya se indicó, en este primer reporte los datos se manejarán en porcentajes, con el propósito de mostrar tendencias en el comportamiento de la muestra. El cuadro 2 resume la información con respecto a la edad de los sujetos que contestaron la encuesta:

CUADRO 2

Edad de los sujetos que contestaron la encuesta

\begin{tabular}{|l|c|}
\hline Edad & $\%$ \\
\hline Menos de 20 años & 0.16 \\
\hline $21-25$ & 0.79 \\
\hline $26-30$ & 50.55 \\
\hline $31-35$ & 30.36 \\
\hline $36-40$ & 11.58 \\
\hline $41-45$ & 4.07 \\
\hline $46-50$ & 1.57 \\
\hline Más de 50 & 0.92 \\
\hline Total & 100.00 \\
\hline
\end{tabular}

Un poco más de la mitad de los sujetos que contestaron la encuesta tienen entre 26 y 30 años de edad. Esto se debe en parte a que para la conformación de la muestra se solicitó la localización de egresados entre estas edades. Lo mismo sucede con el rango siguiente - el segundo en importancia-, cuyas edades fluctúan entre 31 y 35 años. Asimismo, se trata de dos momentos importantes en la trayectoria de los sujetos: inmediatamente después de haber egresado de la carrera y el comienzo del despegue profesional. Por otra parte, el criterio también es congruente con el boom de la comunicación en las universidades 
-finales de la década de los años sesenta y comienzos de la siguiente-, por lo que los egresados ubicados en ambos rangos corresponden a las primeras generaciones de parte de estas instituciones. Por otra parte, esta misma selección incidirá en otras variables, como la actividad profesional y el ingreso.

Esta información se complementa con la sintetizada en el cuadro 3, sobre el género de los sujetos:

CUADRO 3

Género de los sujetos de la muestra

\begin{tabular}{|l|c|}
\hline Género & $\%$ \\
\hline Femenino & 69.73 \\
\hline Masculino & 30.27 \\
\hline Total & 100.00 \\
\hline
\end{tabular}

Como en el caso anterior, estos porcentajes son representativos de la realidad que en este momento se vive en las carreras de comunicación, mismas que de unos años para acá han pasado por un proceso de feminización de su matrícula. Por ello, en trabajos posteriores será importante relacionar estas variables con otras referentes al ejercicio de la profesión.

\section{La titulación}

Como lo indica el cuadro 4, en este caso la muestra presenta un sesgo hacia una titulación elevada por parte de los egresados:

Cuadro 4

Porcentaje de egresados de la muestra con título de licenciatura

\begin{tabular}{|l|c|}
\hline Titulados & $\%$ \\
\hline Si & 47.06 \\
\hline No & 52.94 \\
\hline Total & 100.00 \\
\hline
\end{tabular}

De acuerdo con los datos arrojados en el reporte institucional, el promedio nacional de titulación de las escuelas de comunicación es de aproximadamente $30 \%$. La mayoría de los egresados no pasan por este proceso sobre todo porque la licenciatura en comunicación no está profesionalizada, es decir, no se requiere de título y/o cédula profesional para ejercer. No obstante, instituciones y diversas 
empresas, como Televisa, casas de bolsa e instituciones bancarias, han emprendido programas de titulación para sus empleados, entre los que se encuentran egresados de las carreras de comunicación. Por otra parte, los empleadores siguen sin considerar a la titulación como un elemento importante para contratar egresados de las carreras de comunicación. Más bien lo importante sigue siendo la experiencia profesional. Sin embargo, el optimismo de los datos concentrados en este cuadro se debe en parte a que instituciones como el Instituto Tecnológico y de Estudios Superiores de Monterrey no realizan trabajo de titulación. Asimismo, la investigación producto del área de concentración en la Universidad Autónoma Metropolitana, permite que la mayoría de sus egresados obtengan el título de licenciatura. ${ }^{4}$ Finalmente, la Universidad Iberoamericana ha lanzado un programa de titulación que ha favorecido a un elevado número de egresados, de tal forma que su porcentaje institucional en este rubro se eleva a más de $60 \%$.

\section{La actualización de los egresados}

Más de $75 \%$ del total de sujetos que contestaron la encuesta señalan haber tomado cursos de actualización que van del diplomado al doctorado. Los datos se consignan en el cuadro 5:

CUADRO 5

Modalidades de actualización de los egresados

\begin{tabular}{|l|c|}
\hline Modalidad de actualización & $\%$ \\
\hline Diplomado & 79.38 \\
\hline Especialización & 5.24 \\
\hline Maestría & 12.23 \\
\hline Doctorado & 1.55 \\
\hline Otra licenciatura & 1.55 \\
\hline Total & 100.00 \\
\hline
\end{tabular}

Como puede observarse, el diplomado constituye la principal opción de actualización entre los egresados que contestaron la encuesta. Ello se debe en parte a que en México se ha extendido la oferta educativa en este renglón, como una opción ofrecida por diversas instituciones tanto de educación superior como de actualización profesional. Los diplomados más solicitados son los de merca-

4. De acuerdo con este reporte, una cifra muy reducida de egresados de la Universidad Autónoma Metropolitana no obtiene su título de licenciatura, en la mayoría de los casos, por trámites administrativos que no completan por razones personales. 
dotecnia, docencia y desarrollo humano, seguidos por los de periodismo y comunicación tanto organizacional como institucional

Por otra parte, la especialización es todavía una opción poco solicitada, al menos por los egresados de las licenciaturas en comunicación. Las de fotografía y mercadotecnia constituyen las más solicitadas y en su mayoría se cursaron en el extranjero, particularmente Estados Unidos. En contraparte, $12.23 \%$ de los egresados que contestaron la encuesta señalan haber cursado maestría, la mayoría en comunicación, ya sea sola o en modalidades como organizacional, institucional, desarrollo, etc. Sigue la maestría en administración. En este caso $-y$ posiblemente como tema de una futura investigación sería importante saber si la maestría se cursa por el interés de la actualización o si reviste un carácter remedial, es decir, para cubrir las deficiencias dejadas por la licenciatura. De cualquier manera, cabe señalar que es creciente el número de maestrías que se ofertan en el país, así como el número de aspirantes a cursarlas.

Finalmente, el doctorado y el estudio de otra licenciatura constituyen las opciones menos solicitadas por los egresados de las licenciaturas en comunicación. Los estudios más solicitados son en ciencia política, comunicación audiovisual, didáctica, epistemología, letras, productividad y calidad total, publiciad y tecnología de la educación. La mayoría de estos doctorados se cursaron en el extranjero. Por lo que se refiere a otra licenciatura, la mayoría de las cursadas se relaciona con el arte - cine y teatro-, seguidos por derecho e idiomas.

Para concluir lo referente a la actualización de los egresados, cabe señalar que en buena parte de los casos los estudios realizados tienen estrecha relación con la práctica profesional que desarrollan, ya sea en la actualidad o como trayectoria.

\section{El desempeño profesional}

El cuadro 6 sintetiza los datos sobre el trabajo de los egresados:

CUADRO 6

Situación laboral de los egresados de las carreras de comunicación

\begin{tabular}{|l|c|}
\hline ¿Trabaja en comunicacion? & $\%$ \\
\hline Si & 70.85 \\
\hline No & 29.15 \\
\hline Total & 100.00 \\
\hline
\end{tabular}

Contrariamente a lo que suele manejarse sobre la falta de trabajo para los egresados de las carreras de comunicación, cabe mostrar el elevado porcentaje 
de quienes trabajan profesionalmente en este campo. No obstante, un análisis posterior debe mostrarnos si hay alguna tendencia por regiones o si la demanda de profesionales es más o menos uniforme en las distintas áreas del país en donde se ubican los egresados que contestaron la encuesta. En este sentido, el mayor número de egresados se ubican en las áreas de medios de comunicación, comunicación organizacional y relaciones públicas. Es decir, a grandes rasgos no hay una discriminación entre los campos tradicionales del ejercicio profesional - como los medios de comunicación y las relaciones públicas-y los campos en transformación, como la comunicación organizacional. Asimismo, todavía son pocos los egresados que se desarrollan en los nuevos campos, como la promoción o las comunicaciones electrónicas.

Asimismo, como se verá más adelante, el hecho de contar con un trabajo en comunicación constituye el primer obstáculo por vencer. Sin embargo, para fines de este ànálisis será necesario reflexionar sobre otras variables relacionadas con las satisfacciones inherentes a dicho trabajo, como ingresos, ambiente laboral, etc. Incluso, la posibilidad de que el campo profesional esté abierto no implica necesariamente que hay disponibilidad en las áreas de interés para los egresados, o en las que tradicionalmente se había captado un número importante de los mismos, como el caso creciente de los medios de comunicación.

Por otra parte, el cuadro 7 ilustra la información sobre los egresados que no trabajan en comunicación:

\section{CUADRO 7}

Razones por las que los egresados no trabajan en comunicación

\begin{tabular}{|l|c|}
\hline ¿Por qué no trabaja en comunicacion? & $\%$ \\
\hline No contesta & 31.69 \\
\hline Estoy estudiando & 6.15 \\
\hline Me dedico a la familia & 13.59 \\
\hline No consigo trabajo & 18.77 \\
\hline Perdí mi empleo & 2.91 \\
\hline Otras & 22.01 \\
\hline No válidas & 5.18 \\
\hline Total & 100.00 \\
\hline
\end{tabular}

La interpretación de estos datos es complicada debido a la elevada movilidad laboral de los egresados de las carreras de comunicación. Dicha movilidad se registra en mayor medida al interior de un campo específico (de una agencia de publicidad a otra), aunque también hay casos de movilidad de un campo a otro (de productor de televisión a publirrelacionista). De cualquier manera, 
aproximadamente la tercera parte del cuadro corresponde a sujetos que no respondieron, un aspecto que nuevamente dificulta la interpretación de los datos, pues se trata tanto de encuestas incompletas como de sujetos que no trabajan ni en comunicación ni en otra área; es decir, mujeres que se dedican a su casa y a su familia. Esta cifra engrosaría la correspondiente al rubro "me dedico a la familia" a cerca de $45 \%$.

Por otra parte, aproximadamente $20 \%$ de las respuestas se ubican en los rubros "no consigo trabajo" y "perdí mi empleo" (éste último sorprendentemente bajó a pesar de las fechas en los que se contestó la encuesta). Entre las razones aducidas para no conseguir empleo, la más importante se refiere a no conseguirlo en un campo específico (como puede ser la televisión) o en un lugar definido, como una agencia de publicidad. En estos casos, no encontrar empleo no necesariamente se identifica con una carencia del mismo, sino con la posibilidad creciente de no encontrarlo en un determinado campo, como la televisión. Asimismo, es previsible que quienes están estudiando (6.15\% de los casos) se hayan incorporado gradualmente al mercado laboral. Por último, entre "otras" razones se encuentran el hacerse cargo de un negocio familiar, el obtener mayores ingresos en otros campos, el incursionar en ámbitos desconocidos para buscar mejores ingresos, mejor status, superación profesional, etcétera.

\section{¿En qué trabajan los egresados?}

Como se mencionó en el apartado anterior, posiblemente ésta es una de las principales preguntas que se formulan los actores que participan en el quehacer de la comunicación: docentes, estudiantes, investigadores y empleadores, particularmente. Es también una de las principales directrices que orientan esta investigación, que se desprende de la evolución del campo profesional. Para dar una idea de la distribución de egresados por actividad, fue necesario construir categorías más amplias que las señaladas en las respuestas, además de que se recurrió a las tipologías arrojadas por la evolución de los campos profesionales. Los resultados se presentan en el cuadro 8.

Con respecto a esta sistematización de los datos, y a reserva de ampliarlos en los siguientes apartados, caben las siguientes consideraciones:

a) Desde siempre, el periodismo escrito ha sido una de las principales actividades llevadas a cabo por los egresados, particularmente porque permite llevar a cabo otro tipo de actividades paralelas. Por lo tanto, los resultados arrojados por la encuesta son congruentes con la realidad profesional. Cabe añadir que en este rubro se ha ubicado también a redactores de medios escritos $\mathrm{y}$, en menor número, las actividades relacionadas con el proceso editorial. Asimismo, en el porcentaje elevado de este rubro influye también que en la muestra se incluyen instituciones con una importante tradición en la formación de periodistas, como la Universidad Veracruzana y la Escuela Nacional de Estudios Profesionales Acatlán, de la UNAM. 
CUADRO 8

Principales actividades a las que se dedican los egresados de las carreras de comunicación que respondieron la encuesta

\begin{tabular}{|l|c|}
\hline Actividad & $\%$ \\
\hline Reportero, prensa escrita & 16.67 \\
\hline Audiovisuales & 13.36 \\
\hline Docencia, académicos y catedráticos & 11.94 \\
\hline Comunicación aplicada & 10.85 \\
\hline Publicidad y diseño gráfico & 10.00 \\
\hline Actividades complementarias & 8.80 \\
\hline Empleados & 6.13 \\
\hline Comercio & 4.87 \\
\hline Otras actividades & 3.93 \\
\hline Asesoria, capacitación, consultoría & 2.67 \\
\hline Coordinación & 2.51 \\
\hline Arte & 2.35 \\
\hline No identificables & 2.35 \\
\hline Amas de casa & 2.20 \\
\hline Investigación & 1.79 \\
\hline No respondieron & 1.72 \\
\hline Total & 100.00 \\
\hline
\end{tabular}

b) La segunda actividad en importancia está constituida por los medios audiovisuales. Bajo este rubro se han incluido fotografía, radio, televisión y video, guionismo, locución y producción. En estos tres últimos casos no se identifica el medio al que pertenecen los egresados. Junto con la prensa escrita conformarían el campo profesional de medios de comunicación, considerado como uno de los pioneros en la carrera. La no inclusión en una sola categoría obedece al hecho de que se perdería la perspectiva porcentual tanto de medios audiovisuales como impresos.

c) La docencia es la tercera actividad en importancia. Como se verá, se trata de un espacio que los egresados buscan de manera creciente como modalidad de desarrollo profesional, con diversas posibilidades: de tiempo completo o como una forma de completar sus ingresos, entre otras. ${ }^{5}$ En este caso, es importante

5. Existen, además, otras modalidades de ejercicio de la docencia detectadas en estudios anteriores realizados en México y el extranjero. Por ejemplo, docentes que imparten sus clases en 
señalar que no todos los egresados que se dedican a esta actividad lo hacen en las carreras de comunicación. Hay algunos ubicados en el nivel medio superior e incluso en el nivel medio.

d) La parte aplicada de la comunicación ocupa el cuarto lugar en las actividades realizadas por los egresados. Aquí se incluyeron la comunicación social y organizacional, relaciones públicas y promoción y difusión. Junto con las actividades de publicidad y diseño gráfico constituyen un campo profesional en expansión, al que incluso se le dedica una importancia creciente desde la enseñanza de la comunicación. ${ }^{6}$ Asimismo, se trata de un campo en expansión, pues es creciente su capacidad de contratación en calidad de empleados o free-lancers.

e) Bajo el rubro de área complementaria se han agrupado actividades relacionadas con administración, gerencia y gestión, así como con mercadotecnia. Se trata del espacio profesional en el que más compiten los egresados de comunicación con egresados de otras carreras, particularmente administración y relaciones industriales. Cabe aclarar que aquí también se incluyen, en menor medida, actividades realizadas por egresados relacionadas con puestos directivos en las instituciones en las que prestan sus servicios.

f) Bajo el rubro de empleados se ubican sujetos que no necesariamente realizan actividades de la comunicación, aunque a veces se asumen como tales: empleados bancarios, agentes de viajes, de seguros, capturistas, etc. En todos los casos forman parte de una organización, aunque en la pregunta correspondiente no señalan el puesto que desempeñan. En esta misma categoría están agrupados los analistas, particularmente los que no se ubicaron como pertenecientes a un medio en particular.

g) En las actividades relacionadas con la coordinación el resultado también es heterogéneo, aunque se reduce a tres categorías: la coordinación de producción en medios de comunicación, la coordinación académica en programas de enseñanza a diversos niveles y la coordinación de actividades en oficinas gubernamentales.

h) $\mathrm{La}$ actividad de investigación no necesariamente se relaciona con el quehacer académico, aunque está presente en algunos casos. En otros se vincula con actividades como la comunicación organizacional, la promoción o la mercadotecnia.

más de una institución, con sus consecuencias para la enseñanza y el aprendizaje: desde la repetición de contenidos hasta la dispersión. Seguramente este fenómeno se analizará con más detalle en la cuarta etapa de la investigación, correspondiente a las perspectivas de académicos y alumnos de nuestras instituciones.

6. Prueba de ello es la importancia creciente que los procesos de revisión curricular prestan a esas áreas, mismas que anteriormente sólo habían sido atendidas por un número reducido de escuelas. 


\section{Giro de la organización en la que trabajan los egresados}

La información se resume en el cuadro 9:

\section{CUADRO 9}

Giro de las actividades de la empresa donde trabajan los egresados que contestaron la encuesta

\begin{tabular}{|l|c|}
\hline Giro & $\%$ \\
\hline Educación & 23.18 \\
\hline Gobierno & 16.51 \\
\hline Prensa & 11.59 \\
\hline Servicios & 6.67 \\
\hline Comercio & 6.35 \\
\hline Televisión/Cable & 6.19 \\
\hline Radio & 5.72 \\
\hline Otros & 5.72 \\
\hline Cine & 4.45 \\
\hline Industria editorial & 3.18 \\
\hline Industria & 2.70 \\
\hline No responde & 2.33 \\
\hline Agrícola & 1.89 \\
\hline Casa productora & 1.89 \\
\hline Producción de medios audiovisuales & 1.59 \\
\hline Total & 100.00 \\
\hline
\end{tabular}

Con respecto a esta información caben tres comentarios:

a) El porcentaje más elevado es en educación. Sin embargo, los egresados que se ubican en estas organizaciones no necesariamente se dedican a impartir clases. Las actividades van desde administrativas hasta la promoción y difusión.

b) Existe congruencia entre algunas actividades señaladas por los egresados y el giro de la empresa en que laboran. El caso de la prensa es ilustrativo al respecto, como también lo es el de los medios audiovisuales, particularmente si se hace una suma de todos los giros vinculados con este campo. Sin embargo, esta congruencia no necesariamente se relaciona con actividades propias del campo de la comunicación, como es el caso del comercio. 
c) A primera vista, los datos muestran que el gobierno sigue siendo uno de los principales empleadores de egresados de las carreras de comunicación, con $22.92 \%$. Sin embargo, $69.08 \%$ de las empresas son privadas y únicamente $6.67 \%$ pertenecen al sector servicios. Estos datos corroboran que el sector privado es la principal fuente de empleo de los egresados de las carreras de comunicación, particularmente bajo dos modalidades: el trabajo fijo y el freelance.

\section{Nuevos conocimientos y nuevas habilidades en el campo de la comunicación}

La inserción del egresado en la práctica profesional lo enfrenta a un problema más frecuente de lo que podría suponerse: la confrontación con una realidad distinta a la que aprendió en la escuela y el consecuente cuestionamiento a su formación académica. Es decir, hasta dónde los conocimientos y las habilidades adquiridos en la universidad le permitirán enfrentar el ejercicio de su profesión: una especie de balance que suele poner en desventaja a la institución educativa de la que procede el egresado. Se trata de un ejercicio que realizan todos los egresados, posiblemente de todas las carreras. El documento original -que da cuenta de 1,018 respuestas a este rubro - muestra una gran dispersión al respecto, en buena medida producto de la percepción individual de cada uno de los encuestados y de las necesidades detectadas en su trayectoria profesional. Por limitaciones de espacio se considerarán únicamente los aspectos más relevantes de cada área temática. Esta información se sintetiza en el cuadro 10.

a) El primer lugar está ocupado por los conocimientos no adquiridos en la universidad, con $22.2 \%$, que se relacionan con la práctica profesional del egresado. Entre los más frecuentes se cuentan las diversas modalidades de comunicación (política, interpersonal, oral, social), estadística y matemáticas, materias de ciencias sociales como psicología, sociología y antropología, entre otras. La investigación se reconoce como una herramienta importante, tanto en términos globales como en lo que se refiere a técnicas particulares. En menor medida se alude a conocimientos relacionados con análisis de contenido, particularmente por parte de quienes realizan esta actividad como empleados o free-lancers. Asimismo, se reconoce la necesidad de contenidos que ayuden a contextualizar el análisis de la realidad, como los procesos de globalización e internacionalización de las comunicaciones. 
CUADRO 10

Nuevos conocimientos y nuevas habilidades que, según los egresados, están apareciendo en el campo profesional

\begin{tabular}{||l|c|}
\hline Modalidad & $\%$ \\
\hline $\begin{array}{l}\text { Conocimientos no adquiridos en la } \\
\text { universidad }\end{array}$ & 22.2 \\
\hline Computación & 15.71 \\
\hline Medios de comunicación & 12.57 \\
\hline Práctica & 8.15 \\
\hline Administración, contabilidad y finanzas & 6.68 \\
\hline Mercadotecnia & 4.22 \\
\hline Tecnología & 3.24 \\
\hline Publicidad & 2.94 \\
\hline Comunicación organizacional & 2.55 \\
\hline Relaciones públicas & 2.35 \\
\hline Fotografía & 2.45 \\
\hline Diseño & 2.84 \\
\hline
\end{tabular}

Por otra parte, hay otro conjunto de conocimientos y habilidades que los egresados afirman no haber recibido en la universidad, aunque en sentido estricto pertenecen más bien a la secundaria, a la preparatoria o a un complemento escolar. Es el caso de la falta de dominio de un idioma, particularmente el inglés. Finalmente, se habla también de redacción y ortografía, que son quizá un problema de carácter más personal; una minoría señala la necesidad de haber aprendido mecanografía en la universidad.

b) En segundo lugar, la computación es la habilidad no adquirida en la universidad, reconocida por un mayor número de egresados, $15.71 \%$. Esto se debe en parte a que la encuesta se aplicó a egresados entre 1974 y 1993, es decir, el periodo que abarca el momento en que autores como Marshall McLuhan inician sus planteamientos sobre las perspectivas de la computadora y el momento en que las instituciones de educación superior comienzan a adquirirlas como apoyo a la enseñanza de diversos contenidos. Incluso, en esta última etapa y todavía en la actualidad, salvo algunas instituciones de educación superior, el equipo de cómputo sigue resultando insuficiente para satisfacer las necesidades del alumnado. Por último, cabe destacar que de manera creciente los empleadores solicitan que los futuros profesionales manejen más de un programa, de acuerdo con las funciones y necesidades de la institución de que se trate. Exigencia que no parecen importantes a algunas universidades. 
c) El tercer rubro en orden de relevancia corresponde a los medios de comunicación, con $12.57 \%$. Así, a pesar de ser considerados como campos tradicionales de ejercicio profesional, continúan empleando al mayor número de egresados (5). En este caso la habilidad más importante no adquirida en la universidad se relaciona con el manejo adecuado del equipo para producir cine, radio y televisión, esta relevación proviene de egresados que trabajan en el campo de la producción en medios de comunicación. También se vincula con la falta de habilidades en este tipo de procesos: técnicas de edición, lo más frecuente. Como se verá en su momento, este punto se relaciona con el enlace, el asunto entre teoría y práctica, tan discutida en las universidades. A reserva de retomarlo en su momento, cabe preguntarse si la función de la universidad es capacitar a sus futuros egresados en el manejo de equipo, o privilegiar la reflexión y la formación del pensamiento crítico. Posiblemente no hay una respuesta generalizada, pues en todos los casos tiene que ver con el proyecto de carrera y el perfil del egresado de cada institución. Asimismo, se relaciona con el campo profesional en el que se insertan los egresados, pues las demandas provienen particularmente de quienes se desenvuelven en medios de comunicación y en casas productoras, ya sea como empleados o como free-lancers.

El último punto vinculado a medios de comunicación se refiere a conocimientos y habilidades particulares de cada quien. Por ejemplo, dirección de escena, elaboración de guiones, distribución cinematográfica, etc., hasta sumar un total aproximado de 60 temáticas. Las carencias que aquí pueden encontrarse tienen que ver no sólo con la organización del currículo de cada institución en particular, sino con la selección, por parte del alumno, del área de orientación correspondiente, misma que no siempre se relaciona con la práctica profesional, sino con la temática de conocimientos.

d) Otro aspecto importante destacado por los egresados corresponde a la práctica, con $8.15 \%$ de respuestas, menos de lo que podría pensarse, aunque se relaciona con otras temáticas, específicamente la de medios de comunicación. Viéndolo así, el porcentaje se eleva y queda casi en el nivel de los conocimientos. En este espacio se pretende resolver una discusión que data de hace muchos años y que ahora se complementa con el punto de vista de los egresados. No obstante, la práctica debe reconocerse más como una habilidad que, puede adquirirse fuera de la universidad. Asimismo, la discusión sobre el exceso de teoría y la poca práctica es más propio de recién egresados, y con poco tiempo en el ejercicio profesional.

e) Por otra parte, administración, contabilidad y finanzas suman $6.68 \%$. Las tres temáticas se agrupan pues pertenecen a un tronco común, además de que constituyen conocimientos complementarios a la enseñanza de la comunicación que se incluyen en el plan de estudios en función del perfil del egresado. En los casos en que aparecen, se trata de una necesidad reconocida particularmente por quienes ocupan puestos directivos y posiblemente quienes cuenten con una experiencia profesional más amplia. No sucede lo mismo con la mercadotecnia, que tiene $4.22 \%$ de menciones, pues se trata de un campo en el que se prepara cada vez más a egresados de las carreras de comunicación, también en función 
del perfil del egresado. No obstante, cabe reconocer que desde el principio ha estado muy ligada a la administración, por lo que se trata de un campo en el que coinciden ambos tipos de profesionales. Asimismo, desde la perspectiva del comunicador, la mercadotecnia suele combinarse con otros campos, como la publicidad, la investigación o el ejercicio en medios de comunicación.

f) Con respecto a la tecnología, que tiene $3.24 \%$, egresados encuestados refieren así, como nombre genérico, o bajo sus diversas modalidades, entre las que destaca multimedia. La expresión genérica del término puede ser síntoma de poco conocimiento sobre la temática, mientras que ejemplificar puede mostrar que el entrevistado ha tenido contacto con determinadas tecnologías. A pesar del porcentaje poco significativo, el problema amerita una mayor reflexión, dada su relación con otras temáticas como los conocimientos, medios de comunicación y la propia computación, entre otros. ${ }^{7}$ Finalmente, la problemática de la enseñanza de la comunicación se completa con la irrupción gradual e irremediable de las nuevas tecnologías y las telecomunicaciones en la vida cotidiana de la sociedad. El tema hizo su aparición en foros de discusión pública aproximadamente a principios de la década pasada. Gradualmente y en un número reducido de instituciones, las nuevas tecnologías pasaron al currículo de licenciatura primero como parte de una asignatura y después como materia independiente. Sin embargo, son pocos los casos en que las telecomunicaciones se han abordado desde las perspectivas tanto de formación como del ejercicio profesional del comunicador. Incluso en la mayoría de las instituciones que ofrecen carreras de comunicación y afines, el tema aparece como una pequeña parte del contenido de una materia, a pesar de haber alcanzado dimensiones importantes.

g) Asimismo, hay un número de temáticas con porcentajes poco significativos, a pesar de que constituyen campos que de manera creciente emplean egresados de carreras de comunicación: publicidad (2.94\%), comunicación organizacional $(2.55 \%)$, relaciones públicas $(2.35 \%)$, fotografía $(2.45 \%)$ y diseño, $(2.84 \%)$. Cabe añadir que hasta hace poco las tres primeras, ocupaban un lugar secundario en los planes de estudio de las licenciaturas, como áreas de orientación. En la actualidad, la tendencia de las universidades a acercarse al mercado laboral del comunicador ha propiciado un giro hacia la formación de estudiantes en estos campos. Hay que destacar que, más que en otros casos, las demandas laborales son satisfechas no sólo por egresados de las carreras de comunicación, sino también de relaciones industriales, administración de empresas, etc. Por lo que se refiere a la fotografía, su papel en los planes de estudio se relaciona con el proyecto de carrera, pues hay quienes la conciben como la base de otras modalidades de comunicación visual - cine, televisión y video- y quienes la

7. Las siguientes dos partes del trabajo integran el artículo de Claudia Benassini, "Acercamiento a la evolución del campo profesional de la comunicación desde la perspectiva de sus egresados", por publicarse en María Antonieta Rebeil Corella y Celia Ruizsandoval, (comps.), El poder de las organizaciones, Universidad Iberoamericana-Universidad Latinoamericana, México. 
consideran un complemento a la formación del comunicador, entre otras variantes. Por último, el diseño, ligado profesionalmente sobre todo a ciertas áreas de la publicidad, a la imagen corporativa, etc., se considera como un campo complementario al de la comunicación.

h) Finalmente, en el rubro "varios" (14.1\%), se agrupan tanto algunas temáticas confusas (capitalista, compaginación de intereses, etc.), como otras que por ser tan particulares difícilmente podían agruparse en alguna categoría, además de que en la mayoría de estos casos cada uno fueron contestados únicamente por una persona. Por ejemplo, compras, creatividad (que puede aplicarse a todos los campos profesionales), didáctica, estilo, habilidades, etc. Lo numeroso y diversificado de este rubro da cuenta nuevamente de la dispersión de los resultados producto de la experiencia profesional de cada egresado.

\section{Nuevas funciones en el ejercicio profesional del comunicador}

La inserción del egresado en la profesión lo enfrenta en mayor o menor medida con la evolución del campo. A su vez, este enfrentamiento le proporciona un panorama del rumbo que están tomando las actividades relacionadas con su trabajo, mismas que visualizará con cierta precisión, dependiendo de la experiencia y del acercamiento a ellas. A continuación se presenta un condensado de las 841 respuestas más importantes de acuerdo con la muestra de 668 egresados, con respecto a las nuevas funciones que están apareciendo en el campo de la comunicación. Los datos al respecto se sintetizan en el cuadro 11.

a) En primer lugar, con $28.41 \%$ de las respuestas están las funciones relacionadas con nuevas tecnologías de comunicación. En este caso, las respuestas más frecuentes tienen que ver con multimedia e Internet. Como en el caso anterior, es frecuente encontrar el genérico "tecnología" o "nuevas tecnologías", respuestas provenientes de egresados con menos experiencia profesional o con menos contacto con estos instrumentos. Asimismo, en menor medida hay diversas temáticas, que se vinculan particularmente a la práctica profesional específica de cada egresado. Entre otros casos cabe citar realidad virtual, comunicación vía satélite, fibra óptica, hipertexto, redes de computadora, etcétera.

b) Aunado a este punto, la computación es considerada en segundo lugar como una nueva función dentro del campo profesional de la comunicación, $(15.1 \%)$. En este rubro se ubican las respuestas que genéricamente aluden a la computadora y sus accesorios como un instrumento de trabajo con importancia creciente. Algo que posiblemente sucede en otros campos profesionales.

c) En tercer lugar están las funciones relacionadas con los medios de comunicación $(6.65 \%)$. Sobresalen las vinculadas a la producción audiovisual - particularmente la edición y la animación por computadora-y las que tienen que ver 
con cada medio específico. Como en el caso anterior, esta percepción procede fundamentalmente de egresados con experiencia profesional en este campo.

\section{CUADRO 11}

Nuevas funciones en el ejercicio del comunicador, según los egresados de las carreras

\begin{tabular}{||l|c|}
\hline Función reconocida & $\%$ \\
\hline Nuevas tecnologias de comunicación & 28.41 \\
\hline Computación & 15.1 \\
\hline Medios de comunicación & 6.65 \\
\hline Comunicación organizacional & 5.82 \\
\hline Mercadotecnia & 5.23 \\
\hline Publicidad & 3.56 \\
\hline Investigación & 2.73 \\
\hline Promoción & 2.62 \\
\hline Repercusiones en el ejercicio profesional & 2.62 \\
\hline Diseño & 2.49 \\
\hline Administración & 1.78 \\
\hline Fotografia & 1.18 \\
\hline Globalización & 1.3 \\
\hline Modalidades comunicativas & 1.78 \\
\hline Repercusiones curriculares & 2.14 \\
\hline Otras respuestas & 16.59 \\
\hline
\end{tabular}

d) La comunicación organizacional ocupa el cuarto lugar de las funciones $(5.82 \%)$, seguida de la mercadotecnia $(5.23 \%)$. Con respecto a la primera, caben las consideraciones hechas al hablar de los conocimientos y las habilidades: hasta hace poco, ocupaba un lugar secundario en los planes de estudio de las licenciaturas, como área de orientación. Dado el acercamiento de las universidades al mercado laboral, en la actualidad comienzan a prepararse egresados en estos campos. Es importante insistir que se trata de un campo cuyas demandas laborales son satisfechas tanto por egresados de las carreras de comunicación, como por los de relaciones industriales, administración de empresas, etc. Por su parte, la mercadotecnia hasta hace poco se consideraba como un campo específico de los administradores. Sin embargo, su relación con algunos ámbitos de la carrera como la publicidad y la promoción, particularmente lo relacionado con comportamiento del consumidor, la han colocado como uno de los campos complementarios más promisorios para el ejercicio profesional. 
e) La publicidad ocupa el siguiente lugar (3.56\%) y las opiniones abarcan las diversas modalidades de ejercicio profesional, desde la agencia hasta la producción de comerciales y el free-lance cuando ello es posible. Como en otros casos, se trata de un campo para el que en principio se preparaban egresados en escuelas particulares, aunque las universidades públicas comienzan a hacerlo de manera creciente (8). Por su parte, las relaciones públicas ocupan $2.85 \%$. Llama la atención una percepción de este tipo, pues se trata de uno de los campos profesionales en el que se insertaron egresados de las primeras generaciones de comunicadores, a no ser que se le visualice en su calidad de campo en evolución, es decir, que para subsistir ha tenido que replantear sus objetivos iniciales.

f) El siguiente lugar lo ocupa la investigación (2.73\%), función que puede ligarse con la mercadotecnia, pues algunos egresados hacen este enlace. En menor medida se encuentran respuestas relacionadas con técnicas y con la importancia creciente de la investigación por encuestas en los diagnósticos de climas laborales, políticos, etc. La promoción continúa la lista $(2.62 \%)$, un campo emergente cuya importancia apenas comienza a visualizarse. En este caso se habla particularmente de ventas, programas de imagen y asesoría, por parte de quienes han incursionado en esta actividad.

g) El mismo porcentaje representa las repercusiones en el ejercicio profesional, sobre las que se argumentan de diversas maneras. Se alude tanto a la especialización y la ampliación del campo como a la multidisciplinariedad y la ampliación del campo profesional: posiciones a primera vista contradictorias si no se interpretan en función de la experiencia profesional del egresado. También se plantean nuevas formas de trabajar, como la posibilidad de que los comunicadores se desempeñen a manera de free-lancers, o en calidad de asesores o gestores de proyectos. Finalmente, llama la atención que también se visualiza el reemplazo del publirrelacionista por el comunicador organizacional, a no ser que, como ya se comentó en su momento, se hable de este último campo en la forma original bajo la que fue concebido y en la que se insertaron muchos egresados.

h) Por otra parte, hay un conjunto de funciones que son complemento del ejercicio profesional del comunicador, como diseño $(2.49 \%)$, administración $(1.78 \%)$ y fotografía $(1.18 \%)$. Llama la atención que se perciban como nuevas funciones en el campo de la comunicación, cuando han acompañado a la profesión desde hace por lo menos dos décadas. De aquí la importancia de realizar un seguimiento de la trayectoria del egresado.

i) Como puede observarse hasta aquí, salvo el punto relacionado con las repercusiones en el ejercicio profesional, el resto de las "nuevas" funciones percibidas por los egresados tienen un cariz bastante tradicional. Incluso llama la atención el hecho de que parte de estas funciones datan por lo menos de dos décadas antes y todavía hay quienes las perciben como nuevas. No obstante, hay un número reducido de egresados (apenas $1.3 \%$ ) que perciben nuevas funciones a la luz de la globalización de las comunicaciones: apertura del mercado mundial, internacionalización del mercado y nuevas formas de organización para el ejercicio profesional, entre otras modalidades. Se trata de un punto al que habrá que prestar mayor atención en trabajos posteriores, para conocer su evolución, 
pues el porcentaje en este momento es muy poco significativo. Lo mismo sucede con las funciones relacionadas con modalidades comunicativas $(1.78 \%)$, mismas que, como en otros casos, repercutirán en formas de organizarse para el ejercicio profesional.

j) Finalmente, cabe señalar el caso de las repercusiones curriculares, (2.14\%), entre las que cabe citar la necesidad de mayor actualización, las modificaciones de planes de estudio de licenciatura y mayor especialización. Como puede observarse, se trata de conceptos muy generales, por lo que es de suponerse que la percepción del egresado al respecto es todavía poco clara.

k) El $16.59 \%$ restante corresponde a respuestas que por su dispersión fue imposible agrupar en alguna de las categorías descritas: idiomas, contabilidad y finanzas, elaboración de presupuestos, diseño de estrategias (gubernamentales y en general), planeación, etc. Esta dispersión en parte es producto de la ampliación del campo profesional, misma que propicia que el egresado responda en función de su experiencia, relacionada particularmente con el trabajo al que se dedica en el momento de contestar la encuesta.

\section{Relevancia de la carrera estudiada}

En concordancia con otros datos arrojados por los cuestionarios, la relevancia académica de los estudios universitarios se evalúa en relación con el ejercicio profesional de los egresados. El cuadro 12 resume los datos al respecto:

CUADRO 12

Relevancia de la carrera estudiada

\begin{tabular}{|l|c|}
\hline Características & $\%$ \\
\hline Muy relevante & 32.39 \\
\hline Relevante & 43.00 \\
\hline Poco relevante & 13.86 \\
\hline Irrelevante & 2.83 \\
\hline No responde & 7.92 \\
\hline
\end{tabular}

Como ya se indicó arriba, el reconocimiento de la formación académica está relacionado con el ejercicio profesional. Así, quienes por distintas razones se desarrollan en otro tipo de actividades ajenas a la comunicación presentan mayor tendencia a no responder o a considerar que la formación académica es irrelevante. Por el contrario, en el extremo superior se encuentran quienes han tenido una trayectoria profesional satisfactoria, tienden a reconocer que la formación académica recibida ha sido relevante. Sin embargo, este dato debe tomarse todavía como provisional, pues es necesario cruzarlo con los resultados 
referentes a la percepción que los egresados tienen acerca de los estudios universitarios.

\section{La gratificación en el ejercicio profesional}

La información al respecto se resume en el cuadro siguiente:

CUADRO 13

Gratificación en el ejercicio profesional

\begin{tabular}{|l|c|c|c|c|c|c|}
\hline Aspecto & $(1)$ & $(2)$ & $(3)$ & $(4)$ & $(5)$ & $(6)$ \\
\hline Autonomia de decisión & 33.03 & 41.55 & 14.35 & 2.09 & 0.75 & 8.23 \\
\hline Conocimientos adquiridos & 33.43 & 43.54 & 13.08 & 1.63 & 0.74 & 7.58 \\
\hline Desarrollo profesional & 29.24 & 39.73 & 17.54 & 4.65 & 0.75 & 8.09 \\
\hline Estabilidad laboral & 21.86 & 39.97 & 22.60 & 6.14 & 0.75 & 8.68 \\
\hline Funciones que ejerce & 30.49 & 44.54 & 13.75 & 2.24 & 0.45 & 8.53 \\
\hline Reconocimiento o prestigio social & 2485 & 41.57 & 17.62 & 5.27 & 1.96 & 8.73 \\
\hline Remuneración económica & 9.64 & 30.42 & 32.23 & 15.81 & 2.96 & 9.04 \\
\hline
\end{tabular}

1) Muy gratificado, (2) Gratificado, (3) Regular, (4) Poco gratificado, (5) Nada gratificado, (6) No responde.

Como en el caso anterior, los comentarios tienen todavía un carácter provisional, pues es necesario cruzarlos con otras variables de la encuesta, como ingreso y trayectoria profesional. Sin embargo, pueden hacerse las siguientes consideraciones:

- En todos los aspectos prevalece la situación de "gratificado". Posiblemente "muy gratificado" sea eligido únicamente por quienes consideran su trayectoria profesional muy exitosa. En el otro extremo llama la atención el bajo porcentaje de egresados que se ubican en la situación de "nada gratificado", mismo que aumenta al hablar de la remuneración económica.

- Autonomía de decisión, conocimientos adquiridos y funciones que ejercen son los rubros más consistentes y ubicados principalmente en las dos primeras columnas. Quizá habría que explorar más esta situación en trabajos futuros sobre el tema, aunque en principio sugiere una estrecha relación entre estas variables y la libertad necesaria para un adecuado ejercicio profesional. 
- Finalmente, como era de esperarse, el rubro "remuneración económica" presenta un comportamiento distinto al resto de las columnas. Por lo tanto, es necesario cruzar esta respuesta con los ingresos para saber si existe correlación entre ambas variables.

\section{Las expectativas}

La percepción que de sí mismos tienen los egresados para cinco años más adelante se resume en este cuadro:

CUADRO 14

Expectativas de los egresados

\begin{tabular}{|l|c|}
\hline Percepción del egresado & $\%$ \\
\hline Dedicado a la misma actividad principal que realiza ahora & 11.7 \\
\hline En un cargo superior dentro de la misma actividad & 30.7 \\
\hline Empleado en otra actividad distinta en el campo de la comunicación & 17.60 \\
\hline Como trabajador independiente & 7.96 \\
\hline Como dueño de su propia empresa & 11.87 \\
\hline En un campo distinto a la comunicación & 4.61 \\
\hline No lo ha pensado & 6.70 \\
\hline Desempleado & 0.28 \\
\hline Varios de los anteriores & 9.08 \\
\hline No responde & 0.56 \\
\hline
\end{tabular}

- Con respecto a esta información es interesante destacar que apenas 7.96\% se ubican como trabajadores independientes, cuando la tendencia del ejercicio profesional del comunicador se orienta hacia allá en diversas modalidades, desde el free-lancer hasta el propietario de agencias de publicidad, compañías de investigación, o el asociado con otros egresados -de comunicación o de otras carreras-, en despachos de servicios profesionales.

- Prácticamente la tercera parte de los egresados se percibe en un cargo superior dentro de la misma actividad que actualmente desempeña. A reserva de revisar su trayectoria profesional, es probable que se trate de profesionales que tienen una carrera ascendente en el campo de la comunicación, misma que les permite esa percepción de su actividad a futuro. 
- Finalmente, son muy pocos los que a futuro se perciben como desempleados, a pesar de que en algunos campos profesionales la expectativa de conseguir empleo ha disminuido, como es el caso de la televisión. En contraparte, hay otros campos que van en aumento y sobre los que el mismo egresado ha explorado muy poco, como son la promoción y el diseño de páginas de Internet.

\section{Conclusiones preliminares}

Hasta aquí los primeros resultados arrojados por la base de datos generada con las respectivas bases de datos de cada institución. Como puede observarse, este primer trabajo todavía tiene un carácter puramente descriptivo, por lo que nos pone frente a retos que, como a lo largo de esta investigación, habremos de solucionar creativamente y en equipo. Entre otros, cabe destacar los siguientes:

- La construcción de un marco teórico al cual puedan someterse los datos arrojados por esta investigación. Ya se han hecho ensayos previos apoyados en el interaccionismo simbólico y la construcción social de la realidad. Esta tarea reviste un carácter urgente, particularmente si se pretende legitimar esta línea de trabajo.

- Un análisis más profundo de la evolución del campo profesional del comunicador y sus perspectivas. Como se recordará, esta parte está considerada en el cuestionario institucional. Sin embargo, para su redacción es necesario tomar en cuenta la perspectiva de los empleadores, quienes, junto con los egresados que ya han acumulado una importante trayectoria profesional, están en posibilidades de aportar más información acerca de la configuración que han seguido los campos tradicionales, los emergentes y aquellos en transición, así como la posible repercusión de este fenómeno en la planeación curricular y en la definición del perfil del egresado.

- Llevar a cabo una reorganización de los datos por parte del CONEICC. Con ello se podrán llevar a cabo diagnósticos regionales de la situación en la que se encuentran los egresados, misma que varía de un lugar a otro, particularmente por las diferencias en la distribución de la oferta laboral. Asimismo, diagnósticos de este tipo deben ser de utilidad al interior de cada vocalía, al menos en términos de información susceptible de analizarse y discutirse para proponer futuras acciones. 
- Es necesario llevar a cabo otros cruces de variables que proporcionen indicadores más claros sobre las tendencias que actualmente reviste el campo profesional de los egresados de la carrera de comunicación. Por ejemplo: si a mayor actualización corresponden mayores ingresos, dónde están ubicados los egresados que perciben mejores ingresos, así como un análisis más profundo de la trayectoria profesional de quienes contestaron la encuesta.

- Por lo que se refiere a la encuesta, los datos arrojados por los rubros "actividad" y "giro" muestran la diversidad de combinaciones que tienen los egresados de la carrera para desarrollarse profesionalmente. En la medida en que los datos aquí consignados proceden de diversas instituciones, es importante que esta consideración pueda añadirse al rubro correspondiente al campo de trabajo del egresado. Como en el caso anterior, la misma encuesta proporciona los elementos suficientes para realizar un primer inventario de campos profesionales en los que se ejerce la profesión del comunicador. 